This item was submitted to Loughborough's Research Repository by the author.

Items in Figshare are protected by copyright, with all rights reserved, unless otherwise indicated.

\title{
Enhancing driving safety and user experience through unobtrusive and function-specific feedback
}

PLEASE CITE THE PUBLISHED VERSION

https://doi.org/10.1145/3131726.3131762

PUBLISHER

ACM

VERSION

AM (Accepted Manuscript)

\section{PUBLISHER STATEMENT}

Permission to make digital or hard copies of all or part of this work for personal or classroom use is granted without fee provided that copies are not made or distributed for profit or commercial advantage and that copies bear this notice and the full citation on the first page. Copyrights for components of this work owned by others than ACM must be honored. Abstracting with credit is permitted. To copy otherwise, or republish, to post on servers or to redistribute to lists, requires prior specific permission and/or a fee. Request permissions from permissions@acm.org.

\section{LICENCE}

In Copyright

\section{REPOSITORY RECORD}

Kunze, Alexander, Steve Summerskill, Russell Marshall, and Ashleigh Filtness. 2019. "Enhancing Driving Safety and User Experience Through Unobtrusive and Function-specific Feedback”. figshare. https://hdl.handle.net/2134/26924. 


\section{Enhancing Driving Safety and User Experience Through Unobtrusive and Function-Specific Feedback}

\section{Alexander Kunze}

Loughborough Design School

Loughborough University

Loughborough, LE11 3TU, UK

A.Kunze@lboro.ac.uk
Russell Marshall

Loughborough Design School

Loughborough University

Loughborough, LE11 3TU, UK

R.Marshall@lboro.ac.uk

\begin{abstract}
Inappropriate trust in the capabilities of automated driving systems can result in misuse and insufficient monitoring behaviour that impedes safe manual driving performance following takeovers. Previous studies indicate that the communication of system uncertainty can promote appropriate use and monitoring by calibrating trust. However, existing approaches require the driver to regularly glance at the instrument cluster to perceive the changes in uncertainty. This may lead to missed uncertainty changes and user disruptions. Furthermore, the benefits of conveying the uncertainty of the different vehicle functions such as lateral and longitudinal control have yet to be explored. This research addresses these gaps by investigating the impact of unobtrusive and function-specific feedback on driving safety and user experience. Transferring knowledge from other disciplines, several different techniques will be assessed in terms of their suitability for conveying uncertainty in a driving context.
\end{abstract}

\section{Author Keywords}

Uncertainty communication, unobtrusive feedback, trust.

\section{CCS Concepts}

-Human-centered computing $\rightarrow$ User interface design; $\mathrm{HCl}$ theory, concepts and models; Interaction techniques; Visualization design and evaluation methods; 


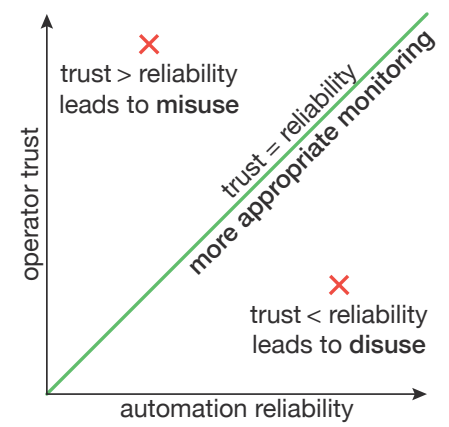

Figure 1: Calibrated trust, i.e. trust that is equal to the system's reliability, promotes appropriate use $[15,27]$
Introduction and Abridged Literature Review Vehicles equipped with automated driving systems (ADSs) promise advances in safety while simultaneously affording users the option to engage in non-driving related tasks (NDRTs). For the foreseeable future, however, users will be required to take over the dynamic driving task (DDT) in critical situations [7]. Such takeover requests (TORs) are signalled when system disengagement is imminent. TORs require users to immediately focus on the driving scene and rapidly comprehend the situation. To prepare users for takeovers, previous research has suggested to constantly communicate uncertainties, quasi the likelihood of TORs.

The existing proposals, however, solely rely on visual information presented in the instrument cluster and thus require users to regularly shift their attention away from the NDRT. This potentially results in missed uncertainty changes and user disruptions. Further, presenting information about the system's uncertainty regarding specific functions, e. g. lateral control, has yet to be explored. Addressing these shortcomings, this research project aims at designing a novel interaction concept to increase safety and experience.

\section{Rationale for Communicating Uncertainties}

When automated systems temporarily relieve users from the DDT, thus taking them out of the loop, significant human factors challenges are the consequence, particularly the Out-of-the-Loop (OOTL) performance problem [6]: When required to take over the previously automated task, human operators are slow to detect and understand problems with the automation and may not be capable of adequately performing the task [5]. Endsley and Kiris [5] ascribe the OOTL performance problem primarily to the user's loss of situation awareness (SA). Analogue to attentional buffers [14], SA can be interpreted as a buffer that is filled when the user focuses on the field relevant for driving (FRD) and is diminished when glancing away from it. Therefore, gaze behaviour is key for enhancing SA and improving safety in takeover scenarios. A factor that is closely connected to the operator's monitoring behaviour is trust. Recent findings indicate that users' trust in the automation and their monitoring frequency are inversely related $[12,26]$. By calibrating the operator's trust (see Figure 1), the appropriate use and monitoring behaviour can be supported. Knowledge about the trustee's ability to perform a certain task forms the basis of trust and providing related information supports the user's trust calibration [13]. Particularly automated systems that utilise neural networks, such as ADSs, allow the dynamic extraction of this information [20].

Uncertainty Communication in the Automotive Domain Several research attempts have focused on providing information about the automation's capability to perform the DDT in order to support the trust calibration of the user. Beller et al. [1] investigated the impact of displaying a schematised uncertain face in the instrument cluster in unclear situations. The results indicate that the communication of system uncertainty increases driving safety, more specifically the time to collision. Further, participants that were provided with the uncertainty information directed their attention in critical situations more to the FRD than the control group, resulting in improved SA.

Helldin et al. [11] explored the communication of seven different uncertainty levels using bars in the instrument cluster, each bar representing one level. The results show that users who were presented with the uncertainty information could afford to allocate their attention away from the FRD and perform NDRTs for a longer time than the control group. Nonetheless, the participants of the experimental group were able to take over the DDT faster than those of the control group. In line with these findings, studies in aviation and the military confirmed the benefits of presenting uncertainty on task performance [4, 8, 20, 31]. 


Key Feedback Aspects
Unobtrusive: Users per-
ceive the system's uncer-
tainty without being disrupted
in the exertion of NDRTs.
Two-Step: The overall un-
certainty is presented to shift
the attention of the user to
the FRD where more detailed
information is available.
Function-Specific: Present-
ing uncertainty concerning
the different parts of the DDT
may aid users with locating
the problem and overcom-
ing the OOTL performance
problem.

The presented publications affirm the outlined benefits of communicating uncertainties. A limitation of the previous work, however, is that users are required to move their focus towards the instrument cluster in order to gain knowledge about the system's current uncertainty. Already, this has shown benefits regarding the practicability of NDRTs [11], but solutions that do not require the driver to glance to the instrument cluster will likely improve this further. An additional limitation is that both publications conveyed the uncertainty of the overall system, not its function-specific uncertainty. Further, the studies did not explore and assess various means of displaying uncertainty in a driving context. Indeed, other researchers proposed to expand the knowledge in this area by exploring different modalities (auditory and visual) and the implications of presenting uncertainty qualitatively, quantitatively, or representationally [24]. Nevertheless, the described shortcomings remain unaddressed and justify the need for more research in the field.

\section{Novel Approach for Conveying Uncertainty}

This project will focus on addressing the three identified gaps by exploring the following research questions:

- How can uncertainty be conveyed without disrupting the user in non-safety critical situations?

- How does the communication of function-specific uncertainty impact driving safety and user experience?

- How can the overall uncertainty and the function-

specific uncertainties be conveyed most intuitively?

To combine the potential benefits of unobtrusive and more detailed communication of uncertainties, a two-step process is proposed: First, the attention of the user is shifted towards the FRD through an unobtrusive stimulus that becomes more salient with increasing uncertainty. In contrast to previous solutions, this allows the user to remain completely engaged in NDRTs as long as the system is fully capable of handling the current situation. Once users shift their attention towards the FRD as a result of an increased salience, function-specific uncertainties will be available within the FRD (step two). It is anticipated that this will allow users to quickly assess which aspect of the DDT is affected and will support them in localising the problem, thus counteracting the OOTL performance problem.

Unobtrusive Feedback

In general, information between system and user can be transferred using the visual (ambient and focal [22]), auditory, and haptic sensory channels [32]. To allow for an unobtrusive and less demanding interaction, an unoccupied channel should be preferred for the presentation of uncertainty information. The auditory and focal visual channel are likely preoccupied with NDRTs such as watching videos or handling mobile devices. This leaves both the haptic channel and ambient vision available for the unobtrusive communication of uncertainty information.

Both sensory channels were shown to be implementable in a driving context. Seat vibration, for instance, has successfully been employed for conveying spatial information about nearby vehicles before takeovers [29, 30]. Similarly, studies have shown that ambient light can be used for comparable applications $[3,16]$. This research project will further explore these channels in the context of communicating uncertainties. Both the general suitability and intuitiveness of haptic and ambient feedback as well as their different instantiations will be investigated. This includes, for instance, the assessment of the suitability of different variables, e. g. light intensity or hue.

Function-Specific Feedback

It is hypothesised that function-specific feedback can help the user to understand which part of the DDT is affected and may counteract the OOTL performance problem. While 


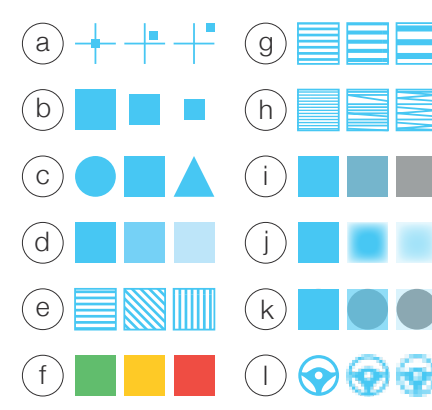

Figure 2: Extended set of visual variables, increasing uncertainty from left to right [19]

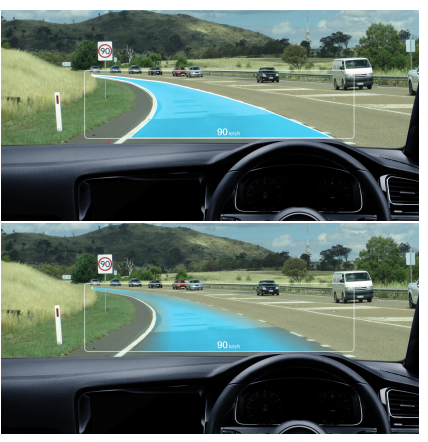

Figure 3: Contact analogue HUD conveying lateral uncertainty through feathered edges (bottom, visual variable $j$ in Figure 2) no related studies have been identified in a driving context, knowledge can be transferred from other domains. In a military context, Neyedli et al. [23] showed that integrating uncertainty information with the affected data re sulted in improvements in reliance compared to a display that presented data and its uncertainty separately. Further, previous studies indicated that graphically displaying uncertainty is (at least) equally effective as numerical or verbal communication [8, 31]. If transferred to a driving context, these findings suggest that graphically integrating the uncertainty information into the environment would be beneficial. For this application, contact analogue head-up displays (cHUDs) could be employed that project augmented reality (AR), world-fixed content onto the driving scene [9, 28].

The integration of uncertainty information with the affected data has been of major interest for Geographic Information Science (GIScience) [18, 25]. A frequently employed strategy within GIScience is to utilise visual variables, manipulable abstract signs, that have initially been identified by Bertin [2] and were subsequently extended [10, 17, 21]. The identified visual variables are: position, size, shape, value, orientation, colour, grain, arrangement, saturation, crispness, transparency, and resolution (see Figure 2, illustrations a-I). In addition to the described abstract signs that vary in a single visual variable, iconic symbols make use of metaphors, such as a clock for depicting time, and might be easier to match with different uncertainty components [19]. MacEachren [19] conducted two linked empirical studies to examine the intuitiveness of the presented abstract as well as additional iconic variables for communicating uncertainty in a geographic context. The results indicate that particularly crispness (j) and location (a) can present uncertainty very intuitively, followed by arrangement $(h)$, value $(d)$, size (b), and transparency (k). Additionally, abstract vehicles led to quicker judgements, while iconic symbols were more accurately judged. This research project will explore the usability of the visual variables in a driving context (see Figure 3 ), identify the most intuitive variables for each DDT function, and subsequently compare the identified variables with the use of more traditional icons and diagrams as employed by Beller et al. [1] and Helldin et al. [11].

\section{General Methodology and Proposed Studies}

This research takes a controlled stepwise approach. First, a key set of haptic and ambient signals which have greatest saliency and intuitiveness for drivers engaged in NDRTs will be identified. In a parallel study, participants will be asked to rate the intuitiveness of different visual variables for conveying function-specific uncertainties (see Figure 3). Informed by step one, these select signals and visual variables will be tested in a dynamic driving environment. A driving simulator has been selected rather than on-road driving to allow for manipulation of the vehicle dashboard in a safe context. This study aims to investigate the impacts of the proposed two-level interaction process on driving safety and user experience. This will include objective measurements of driving performance such as time to collision, braking intensity, and lane deviation as well as measurements of SA and trust, including analyses of the gaze behaviour. As the communication of function-specific uncertainty allows the user to identify the affected aspect of the DDT, further research will explore the practicability of partial takeovers, e.g. performing only the lateral control while the ADS continues to control the longitudinal movement. This new approach to vehicle interaction is anticipated to ultimately enhance the safety of ADSs by keeping users constantly aware of the vehicle's current capabilities and providing information that guides them to the problem at hand. Further, users do not have to regularly monitor the instrument cluster as the information is perceived even when performing NDRTs, thus improving their user experience. 


\section{REFERENCES}

1. Johannes Beller, Matthias Heesen, and Mark Vollrath. 2013. Improving the Driver Automation Interaction: An Approach Using Automation Uncertainty. Human Factors: The Journal of the Human Factors and Ergonomics Society 55, 6 (2013), 1130-1141. DOI : http://dx.doi.org/10.1177/0018720813482327

2. Jacques Bertin. 1967. Semiology of Graphics: Diagrams, Networks, Maps. University of Wisconsin.

3. Shadan Sadeghian Borojeni, Lewis Chuang, Wilko Heuten, and Susanne Boll. 2016. Assisting Drivers with Ambient Take Over Requests in Highly Automated Driving. In Proceedings of the 8th International Conference on Automotive User Interfaces and Interactive Vehicular Applications. ACM, Ann Arbor, MI, USA, 237-244. DOI :

http://dx.doi.org/10.1145/3003715.3005409

4. Mary T. Dzindolet, Scott A. Peterson, Regina A. Pomranky, Linda G. Pierce, and Hall P. Beck. 2003. The role of trust in automation reliance. International Journal of Human Computer Studies 58, 6 (2003), 697-718. DOI :

http://dx.doi.org/10.1016/S1071-5819(03)00038-7

5. Mica R. Endsley. 1995. Toward a Theory of Situation Awareness in Dynamic Systems. Human Factors: The Journal of the Human Factors and Ergonomics Society 37, 1 (1995), 32-64. DOI :

http://dx.doi.org/10.1518/001872095779049543

6. Mica R. Endsley. 2017. From Here to Autonomy: Lessons Learned From Human-Automation Research. Human Factors The Journal of the Human Factors and
Ergonomics Society 59, 1 (2017), 5-27. DOI : http://dx.doi.org/10.1177/0018720816681350

7. European Road Transport Research Advisory Council. 2015. Automated Driving Roadmap. (2015). http://www.ertrac.org/uploads/documentsearch/ id38/ERTRAC

8. Richard Finger and Ann M. Bisantz. 2002. Utilizing graphical formats to convey uncertainty in a decision-making task. Theoretical Issues in Ergonomics Science 2, 1 (2002), 1-25. DOI : http://dx.doi.org/10.1080/14639220110110324

9. Renate Haeuslschmid, Yixin Shou, John O'Donovan, Gary Burnett, and Andreas Butz. 2016. First Steps towards a View Management Concept for large-sized Head-Up Displays with Continuous Depth. In Proceedings of the 8th International Conference on Automotive User Interfaces and Interactive Vehicular Applications. ACM, Ann Arbor, MI, USA, 1-8.

10. Łukasz Halik. 2012. The analysis of visual variables for use in the cartographic design of point symbols for mobile Augmented Reality applications. Geodesy and Cartography 61, 1 (2012), 19-30. DOI : http://dx.doi.org/10.2478/v10277-012-0019-4

11. Tove Helldin, Göran Falkman, Maria Riveiro, and Staffan Davidsson. 2013. Presenting system uncertainty in automotive Uls for supporting trust calibration in autonomous driving. Proceedings of the International Conference on Automotive User Interfaces and Interactive Vehicular Applications AutomotiveUl '13 5 (2013), 210-217. DOI : http://dx.doi.org/10.1145/2516540.2516554 
12. Sebastian Hergeth, Lutz Lorenz, Roman Vilimek, and Josef F. Krems. 2016. Keep Your Scanners Peeled: Gaze Behavior as a Measure of Automation Trust During Highly Automated Driving. Human Factors: The Journal of the Human Factors and Ergonomics Society 58, 3 (2016), 509-519. DOI :

http://dx.doi.org/10.1177/0018720815625744

13. Kevin Anthony Hoff and Masooda Bashir. 2015. Trust in Automation: Integrating Empirical Evidence on Factors That Influence Trust . Human Factors: The Journal of the Human Factors and Ergonomics Society 57, 3 (2015), 407-434. DOI :

http://dx.doi.org/10.1177/0018720814547570

14. Katja Kircher and Christer Ahlström. 2009. Issues related to the driver distraction detection algorithm AttenD. In 1st International Conferences on Driver Distraction and Inattention. Ifsttar, Gothenburg, Sweden, 1-15.

http://trid.trb.org/view . aspx?id=1240063

15. John D. Lee and Katrina A. See. 2004. Trust in Automation: Designing for Appropriate Reliance. Human Factors 46, 1 (2004), 50-80.

16. Andreas Loecken, Wilko Heuten, and Susanne Boll. 2015. Supporting Lane Change Decisions with Ambient Light. In Proceedings of the 7th International Conference on Automotive User Interfaces and Interactive Vehicular Applications. ACM, Nottingham, UK, 204-211. DOI : http://dx.doi.org/10.1145/2799250.2799259

17. Alan M. MacEachren. 1992. Visualizing uncertain information. Cartographic Perspective 13 (1992), 10-19. DOI : http://dx.doi.org/10.1.1.62.285
18. Alan M. MacEachren, Anthony Robinson, Susan Hopper, Steven Gardner, Robert Murray, Mark Gahegan, and Elisabeth Hetzler. 2005. Visualizing geospatial information uncertainty: What we know and what we need to know. Cartography and Geographic Information Sciencelnformation Science 32, 3 (2005), 139-160. DOI :

http://dx.doi.org/10.1559/1523040054738936

19. Alan M. MacEachren, Robert E Roth, James O'Brien, Derek Swingley, and Mark Gahegan. 2012. Visual

Semiotics and Uncertainty Visualisation: An Empirical Study. In IEEE Transactions on Visualization and Computer Graphics, Vol. 18. IEEE, 2496-2505. DOI : http://dx.doi.org/10.1109/TVCG. 2012.279

20. John M. McGuirl and Nadine B. Sarter. 2006. Supporting trust calibration and the effective use of decision aids by presenting dynamic system confidence information. Human Factors 48, 4 (2006), 656-665. DOI :

http://dx.doi.org/10.1518/001872006779166334

21. Joel L. Morrison. 1974. A theoretical framework for cartographic generalization with the emphasis on the process of symbolization. International Yearbook of Cartography 14 (1974), 115-127.

22. National Research Council (US) Committee on Vision. 1985. Emergent techniques for assessment of visual performance. National Academies Press (US), Washington, DC. https://www.ncbi.nlm.nih.gov/books/NBK219039/ 
23. Heather F. Neyedli, Justin G. Hollands, and Greg A. Jamieson. 2011. Beyond Identity: Incorporating System Reliability Information Into an Automated Combat Identification System. Human Factors: The Journal of the Human Factors and Ergonomics Society 53, 4 (2011), 338-355. DOI :

http://dx.doi.org/10.1177/0018720811413767

24. Brittany E. Noah and Bruce N. Walker. 2017. Trust Calibration through Reliability Displays in Automated Vehicles. Proceedings of the Companion of the 2017 ACM/IEEE International Conference on Human-Robot Interaction - HRI '17 (2017), 361-362. DOI :

http://dx.doi.org/10.1145/3029798.3034802

25. Alex T. Pang, Craig M. Wittenbrink, and Suresh K. Lodha. 1997. Approaches to uncertainty visualization. The Visual Computer 13, 8 (1997), 370-390. DOI : http://dx.doi.org/10.1007/s003710050111

26. Raja Parasuraman and Dietrich Manzey. 2010. Complacency and bias in human use of automation: an attentional integration. Human factors 52 (2010), 381-410. DOI :

http://dx.doi.org/10.1177/0018720810376055

27. Raja Parasuraman and Victor Riley. 1997. Humans and Automation: Use, Misuse, Disuse, Abuse. Human Factors The Journal of the Human Factors and Ergonomics Society 39, 2 (1997), 230-253.

28. Lisa Pfannmueller, Martina Kramer, Bernhard Senner, and Klaus Bengler. 2015. A Comparison of Display
Concepts for a Navigation System in an Automotive Contact Analog Head-up Display. Procedia Manufacturing 3 (2015), 2722-2729. DOI : http://dx.doi.org/10.1016/j.promfg.2015.07.678

29. Matti Schwalk, Niko Kalogerakis, and Thomas Maier. 2015. Driver Support by a Vibrotactile Seat Matrix Recognition, Adequacy and Workload of Tactile Patterns in Take-over Scenarios During Automated Driving. Procedia Manufacturing 3 (2015), 2466-2473. DOI :

http://dx.doi.org/10.1016/j.promfg. 2015.07.507

30. Ariel Telpaz, Brian Rhindress, Ido Zelman, and Omer Tsimhoni. 2015. Haptic Seat for Automated Driving : Preparing the Driver to Take Control Effectively. Proceedings of the 7th International Conference on Automotive User Interfaces and Interactive Vehicular Applications (2015), 23-30. DOI : http://dx.doi.org/10.1145/2799250.2799267

31. Lu Wang, Greg A. Jamieson, and Justin G. Hollands. 2009. Trust and reliance on an automated combat identification system. Human factors 51, 3 (2009), 281-291. DOI : http://dx.doi.org/10.1177/0018720809338842

32. Hermann Winner and Stephan Hakuli. 2012. Handbuch Fahrerassistenzsysteme (2nd ed.). Vieweg+Teubner Verlag, Wiesbaden. DOI :

http://dx.doi.org/10.1007/978-3-8348-8619-4 